\title{
Multi-linear sparse reconstruction for SAR imaging based on higher-order SVD
}

\author{
Yu-Fei Gao ${ }^{1}$, Guan Gui ${ }^{2}$ (D), Xun-Chao Cong ${ }^{1}$, Yue Yang ${ }^{1}$, Yan-Bin Zou ${ }^{1}$ and Qun Wan ${ }^{*}$
}

\begin{abstract}
This paper focuses on the spotlight synthetic aperture radar (SAR) imaging for point scattering targets based on tensor modeling. In a real-world scenario, scatterers usually distribute in the block sparse pattern. Such a distribution feature has been scarcely utilized by the previous studies of SAR imaging. Our work takes advantage of this structure property of the target scene, constructing a multi-linear sparse reconstruction algorithm for SAR imaging. The multi-linear block sparsity is introduced into higher-order singular value decomposition (SVD) with a dictionary constructing procedure by this research. The simulation experiments for ideal point targets show the robustness of the proposed algorithm to the noise and sidelobe disturbance which always influence the imaging quality of the conventional methods. The computational resources requirement is further investigated in this paper. As a consequence of the algorithm complexity analysis, the present method possesses the superiority on resource consumption compared with the classic matching pursuit method. The imaging implementations for practical measured data also demonstrate the effectiveness of the algorithm developed in this paper.
\end{abstract}

Keywords: Spotlight SAR imaging, Kronecker constraint, Compressed sensing, HOSVD, Alternating least squares

\section{Introduction}

Synthetic aperture radar (SAR) is an important detection system in many fields for decades, such as remote sensing, environmental monitoring, and ground mapping [1-4]. Imaging algorithm is the key point of SAR application, which extracts the scatter coefficients of targets and reconstructs the image of target scene from the radar echoes $[5,6]$. On the azimuth direction, a larger aperture can be synthesized by the coherent processing and relative motions between the antenna and targets. For the range direction, the pulse compression is usually applied to obtain a good range resolution. However, the existing methods encounter several challenges in practical applications. For instance, the overwhelming amount of resource requests for storing and operating in receiver since the high sampling rate; in addition, the conventional imaging method, such as polar format algorithm (PFA), suffers from the sensibility to sidelobe disturbance, which leads to it being unsuitable for high-resolution imaging. For

\footnotetext{
*Correspondence: wanqun@uestc.edu.cn

'School of Electronic Engineering, University of Electronic Science and

Technology of China, No. 2006, Xiyuan Ave., West Hi-Tech Zone, Chengdu 611731, China

Full list of author information is available at the end of the article
}

solving the problem of high demand on hardware, the compressed sensing (CS) framework has been introduced into SAR imaging [7-11], which can extract necessary information at a lower sampling rate than Nyquist limit [12-14]. Furthermore, the CS-based approach is able to accomplish SAR imaging with the low sidelobe and possess the robustness to noise [15-20]. But the existing methods only utilize the spatial sparsity of the observation without taking advantage of structural features of target scene. In 2016, a sparse reconstruction SAR imaging method was proposed by Zhao et al., which employed structured sparsity constraint based on Bayesian learning framework [21]. But this method requires rigid conditions to ensure model matching; consequently, the imaging quality may be worse if observations cannot perfectly match the model selected.

For practical applications of SAR imaging, the scatterers generally tend to be clustered together as blocks. In this paper, a tensor-decomposition-based algorithm is developed to exploit such block sparsity of targets. Tensor decomposition is a kind of higher-order data processing framework which can make use of the multidimensional and highly structured features in signal [22-24]. Recently, combining tensor modeling with sparse reconstruction

\section{Springer Open}

(c) The Author(s). 2017 Open Access This article is distributed under the terms of the Creative Commons Attribution 4.0 International License (http://creativecommons.org/licenses/by/4.0/), which permits unrestricted use, distribution, and reproduction in any medium, provided you give appropriate credit to the original author(s) and the source, provide a link to the Creative Commons license, and indicate if changes were made. 
is becoming the focus of attention in signal processing. In 2010, Lim and Comon proposed a sparse reconstruction method based on low-rank tensor decomposition and analyzed the condition of uniqueness for decomposition [25]. In 2011, Duarte and Eldar introduced the Kronecker structure into dictionary constructing, proposing the Kronecker-CS framework [26]. In 2012, Sidiropoulos and Kyrillidis investigated CS theorem with regard to low-rank tensor signal processing, proposing a two-step sparse reconstruction algorithm [27]. In 2013, Caiafa and Cichocki proposed two types of matching pursuit algorithms based on tensor decomposition [28]. However, there has been little work in SAR imaging based on sparse reconstruction combined with tensor decomposition. The higher-order CS approach in hyperspectral imaging enlightens our study in signal modeling $[26,29,30]$. In this paper, an imaging algorithm is developed based on higher-order singular value decomposition (HOSVD) $[31,32]$ with the Kronecker constraint of reflected waves, which can take advantage of the block sparse feature of target scene to gain more robust high-resolution imaging performance. The theoretical analysis and simulation experiments verify that the developed algorithm is superior to the reference methods especially in severe conditions, which demonstrates the practical significances.

The following is the organization of the rest in this paper: Section 2 illustrates the signal model for spotlight SAR; Section 3 introduces the tensor modeling based on HOSVD; Section 4 proposes the algorithm by introducing the multi-linear block sparse reconstruction and the preprocessing scheme for dictionary construction; Section 5 demonstrates several numerical simulations to verify the effectiveness of the proposed algorithm; Section 6 concludes this paper.

\section{Problem formulation}

First of all, several notions of this paper are stated as follows.

- The scalar is indicated by italic letter, e.g., $x$.

- The vector and matrix are indicated by bold italic letters, e.g., $\boldsymbol{x}, \boldsymbol{X}$.

- The tensor is indicated by calligraphic capital letter, e.g., $\mathcal{X}$.

- The index of a set is indicated by script letter, e.g., $\mathscr{X}$.

- $\|\boldsymbol{x}\|_{0}$ is the $l_{0}$-norm which indicates the number of nonzero elements in $\boldsymbol{x}$.

- $\|\mathcal{X}\|_{F}$ is the Frobenius norm of the tensor $\mathcal{X}$, denoting the square root of the sum of the absolute squares of its elements.

- The operator $\otimes$ denotes the Kronecker product between matrices.

- The operator $\times_{n}$ denotes the mode- $n$ product between tensor and matrix, where mode is considered as the order of tensor data [33].
- For a third-order tensor $\mathcal{X} \in \mathbb{C}^{M \times N \times P}$, define $\boldsymbol{X}_{(1)}$ as a rectangular matrix combined all $P$ slices of $\mathcal{X}$ under mode-1, i.e., $\boldsymbol{X}_{(1)}=\left[\boldsymbol{X}_{1}, \boldsymbol{X}_{2}, \ldots, \boldsymbol{X}_{P}\right]^{T} \in \mathbb{C}^{(P N) \times M}$; similarly, $\boldsymbol{X}_{(2)}$ and $\boldsymbol{X}_{(3)}$ denote the unfolded matrix of $\mathcal{X}$ along mode- 2 and mode-3, respectively.

This paper considers spotlight SAR for capturing the structural information of the SAR targets, which is a beamforming-based observation system [34-36]. The radio beam will be pointed to the observation scene continuously while the aircraft carrying SAR flying cross over the target scene. The geometry of such model is shown in Fig. 1. $\theta$ and $\phi$ indicate the azimuth and pitch angles of radio wave, respectively. $R\left(x_{0}, y_{0}, z_{0}\right)$ represents the distance between the antenna and target at $\left(x_{0}, y_{0}, z_{0}\right) ; R_{c}$ represents the distance between the antenna and the center of the scene. Defining the speed of light as $c$, then the corresponding propagation time can be written as $\tau_{c}=2 R_{c} / c$.

The received signal can be represented as

$$
y(t, \theta, \phi)=\sum_{x, y, z} g(x, y, z) s(t-\tau(x, y, z))+e(t),
$$

where $g(x, y, z)$ is the scattering function of the scatterer located at $(x, y, z) ; s(t)$ is the autocorrelation function of transmitted signal; $e(t)$ is the additive noise. For the simplicity, the noise item is not shown in the following content. After transformation to frequency domain, the phase history of the received signal can be represented as

$$
Y(f, \theta, \phi)=C(f) \sum_{x, y, z} g(x, y, z) \exp \left(H_{x, y, z}(f, \theta, \phi)\right),
$$

where $H_{x, y, z}(f, \theta, \phi)=-j \frac{4 \pi}{c}(x f \cos \theta \cos \phi+y f \cos \theta$ $\sin \phi+z f \sin \theta)$ is the phase factor. The constant term $C(f)=S(f) \exp \left(-j 2 \pi f \tau_{c}\right)$ can be ignored in the following discussion, where $S(f)$ is the Fourier transform of $s(t)$.

Because the parameters in the phase factor of the signal model (2) are coupled, it is needed to implement an interpolation approach for decoupling [34]. Thus, the coordinate conversion is done from polarization to Cartesian system [37]. Assume there are $M_{1}, M_{2}, M_{3}$ grid points in the directions of the axis of $\mathrm{x}, \mathrm{y}, \mathrm{z}$, respectively, and the numbers of grid points for $f, \theta, \phi$ are $P_{1}, P_{2}, P_{3}$, respectively. Then, the steering vector of the scatterer located at $\left(x_{j}, y_{k}, z_{l}\right)$ is

$$
\begin{aligned}
\boldsymbol{d}_{j, k, l}= & {\left[d_{j, k, l}(1,1,1), \ldots, d_{j, k, l}\left(P_{1}, P_{2}, 1\right), \ldots,\right.} \\
& \left.d_{j, k, l}\left(1,1, P_{3}\right), \ldots, d_{j, k, l}\left(P_{1}, P_{2}, P_{3}\right)\right]^{T},
\end{aligned}
$$

where $d_{j, k, l}\left(p_{1}, p_{2}, p_{3}\right)=\exp \left(-j \frac{4 \pi}{c}\left(x_{j} u_{p_{1}}+y_{k} v_{p_{2}}+\right.\right.$ $\left.\left.z_{l} w_{p_{3}}\right)\right)$. The steering matrix $\boldsymbol{D} \in \mathbb{C}^{P \times M}$ is defined as $\left[\boldsymbol{d}_{1,1,1}, \ldots, \boldsymbol{d}_{M_{1}, M_{2}, 1}, \ldots, \boldsymbol{d}_{1,1, M_{3}}, \ldots, \boldsymbol{d}_{M_{1}, M_{2}, M_{3}}\right]$, where 


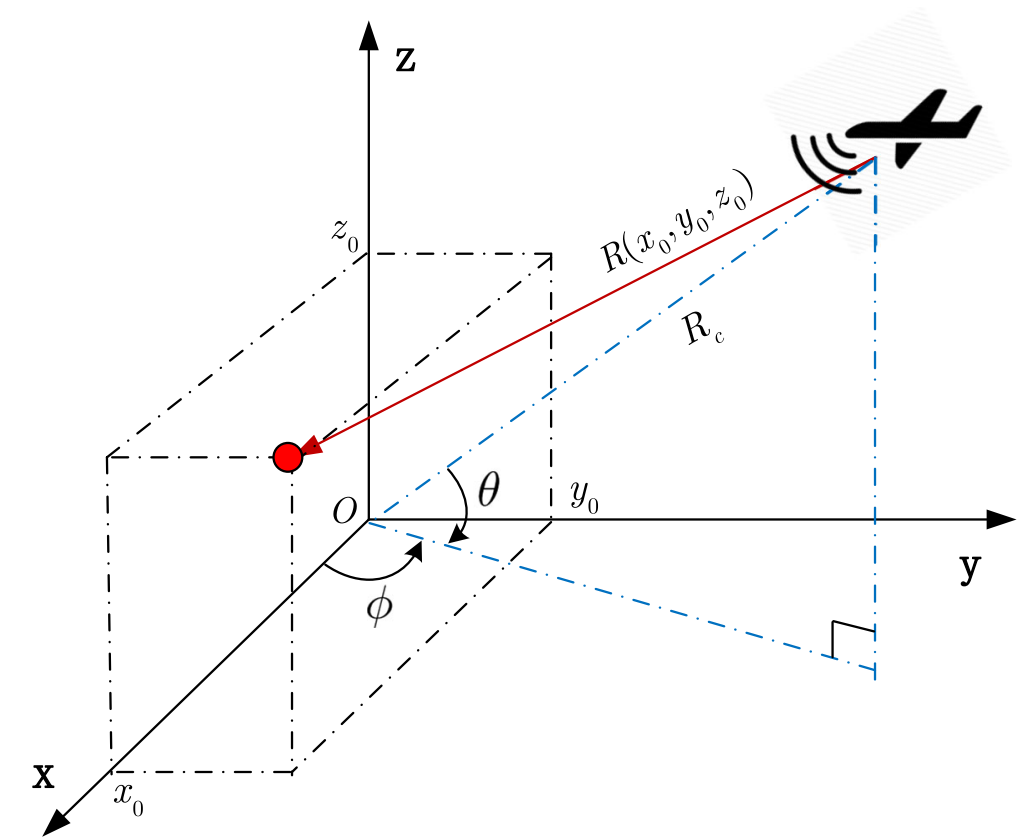

Fig. 1 The geometry of spotlight SAR for point scatterers

$M=M_{1} \times M_{2} \times M_{3}, P=P_{1} \times P_{2} \times P_{3}$. Hence, the received signal model (2) can be written as

$$
y=D s,
$$

where $\boldsymbol{s}=\left[g\left(x_{1}, y_{1}, z_{1}\right), \ldots, g\left(x_{M_{1}}, y_{M_{2}}, z_{1}\right), \ldots, g\left(x_{1}, y_{1}, z_{M_{3}}\right)\right.$ $\left., \ldots, g\left(x_{M_{1}}, y_{M_{2}}, z_{M_{3}}\right)\right]^{T}$. Without loss of generality, considering the $\left(p_{1} p_{2} p_{3}, m_{1} m_{2} m_{3}\right)$-th element of matrix $\boldsymbol{D}$ is $\exp \left(-j \frac{4 \pi}{c}\left(x_{m_{1}} u_{p_{1}}+y_{m_{2}} v_{p_{2}}+z_{m_{3}} w_{p_{3}}\right)\right)$, thus $\boldsymbol{D}=$ $D_{3} \otimes D_{2} \otimes D_{1}$, by which the Kronecker structure can be shown explicitly. As a consequence, the received signal can be rewritten as

$$
y=\left(D_{3} \otimes D_{2} \otimes D_{1}\right) s
$$

\section{Tensor decomposition for SAR imaging}

In signal processing field, a tensor indicates the multilinear data with more than two modes. This paper adopts the HOSVD [24, 31], which can be represented as

$$
\mathcal{Y}=\mathcal{S} \times{ }_{1} D_{1} \times{ }_{2} D_{2} \times{ }_{3} \ldots \times_{N} D_{N}
$$

where $\mathcal{Y}$ denotes the received signal tensor. For a HOSVD, the operation criterion is

$$
\min _{\mathcal{S},\left\{\boldsymbol{D}_{n}\right\}_{n=1}^{N}}\left\|\mathcal{Y}-\mathcal{S} \times{ }_{1} \boldsymbol{D}_{1} \times{ }_{2} \boldsymbol{D}_{2} \times_{3} \ldots \times_{N} \boldsymbol{D}_{N}\right\|_{F}^{2} .
$$

The one-dimensional expansion of a tensor is stacking all elements along the mode-1, i.e., $\boldsymbol{y}=\operatorname{vec}\left(\boldsymbol{Y}_{(1)}\right)$. Thus, (6) can be rewritten as

$$
\boldsymbol{y}=\left(\boldsymbol{D}_{N} \otimes \boldsymbol{D}_{N-1} \otimes \ldots \otimes \boldsymbol{D}_{1}\right) \boldsymbol{s} .
$$

The equation above indicates that the HOSVD of a tensor signal is equivalent to a linear representation with Kronecker dictionary. It is defined that a $K$-sparse representation of signal $\boldsymbol{y}$ is existed on a dictionary $\boldsymbol{D}$, if the following condition is true:

$$
\boldsymbol{y}=\boldsymbol{D} \boldsymbol{s}, \quad \text { s.t. }\|\boldsymbol{s}\|_{0} \leqslant K
$$

where $K$ is named as sparsity. Because the rows of dictionary is less than the columns, this is an underdetermined system; hence, no unique solution of $s$ exists. However, if the signal possesses sparsity, a solution may be obtained under the following condition [38],

$$
K<\frac{1}{2}\left(1+\frac{1}{\mu(D)}\right)
$$

where $\mu(\boldsymbol{D})=\max _{i \neq j}\left|\left\langle\boldsymbol{d}_{i}, \boldsymbol{d}_{j}\right\rangle\right|$, representing the coherence coefficient of dictionary $\boldsymbol{D}$; here, $\left\langle\boldsymbol{d}_{i}, \boldsymbol{d}_{j}\right\rangle$ denotes the inner product of $\boldsymbol{d}_{i}$ and $\boldsymbol{d}_{j}$. When the dictionary possesses Kronecker structure, i.e., $\boldsymbol{D}=\boldsymbol{D}_{N} \otimes \boldsymbol{D}_{N-1} \otimes$ $\ldots \otimes D_{1}$, the coherence coefficient should be $\mu(D)=$ $\max \left\{\mu_{1}, \mu_{2}, \ldots, \mu_{N}\right\}$, with $\mu_{n}=\mu\left(\boldsymbol{D}_{n}\right), n=1,2, \ldots, N$. It is demonstrated that the total coherence depends merely on the factor matrix with the largest coherence coefficient for a Kronecker dictionary [39, 40].

Definition 1 (Multi-linear block sparsity) $A$ tensor $\mathcal{Y}$ is defined as $\left(K_{1}, K_{2}, \ldots, K_{N}\right)$-block sparse, if only 
$K_{n}(n=1, \ldots, N)$ columns in the factor matrices of its HOSVD need to be computed, i.e.,

$$
\begin{aligned}
& \mathcal{Y}=\mathcal{S} \times{ }_{1} \boldsymbol{D}_{1} \times{ }_{2} \boldsymbol{D}_{2} \times{ }_{3} \ldots \times_{N} \boldsymbol{D}_{N}, \\
& \text { s.t. } \quad s_{i_{1}, i_{2}, \ldots, i_{N}}=0 \forall\left(i_{1}, i_{2}, \ldots, i_{N}\right) \notin\left\{\mathscr{I}_{1}, \mathscr{I}_{2}, \ldots, \mathscr{I}_{N}\right\},
\end{aligned}
$$

where $\mathscr{I}_{n}=\left[i_{n}^{1}, i_{n}^{2}, \ldots, i_{n}^{K_{n}}\right]$ represents a subset of index for mode- $n(n=1,2, \ldots, N)$.

The above definition demonstrates that the nonzero elements of core tensor are included in a subtensor $\mathcal{S}\left(\mathscr{I}_{1}, \mathscr{I}_{2}, \ldots, \mathscr{I}_{N}\right)$. Here, $\mathscr{I}_{n}=\left\{i_{n}^{1}, i_{n}^{2}, \ldots, i_{n}^{K_{n}}\right\}$ is a subset of the index set for mode- $n(n=1,2, \ldots, N)$. For instance, given a tensor $\mathcal{X} \in \mathbb{C}^{5 \times 5 \times 5}, \mathscr{I}_{1}=\mathscr{I}_{2}=\mathscr{I}_{3}=$ $\{2,4\}$, then $\mathcal{Y}=\mathcal{X}\left(\mathscr{I}_{1}, \mathscr{I}_{2}, \mathscr{I}_{3}\right)$ is a third order tensor with a size of $2 \times 2 \times 2$. And the first and second elements along each mode of $\mathcal{Y}$ are the second and fourth elements along each mode of $\mathcal{X}$, respectively, i.e., $\mathcal{Y}$ picks the corresponding elements in $\mathcal{X}$ indexed by $\left\{\mathscr{I}_{n}\right\}_{n=1}^{3}$. As a consequence, $\boldsymbol{y}$ is $K$-sparse with regard to Kronecker dictionary $\boldsymbol{D}=\left(\boldsymbol{D}_{N} \otimes \boldsymbol{D}_{N-1} \otimes \ldots \otimes \boldsymbol{D}_{1}\right)$, where $K=$ $K_{1} K_{2} \ldots K_{N}$. It should be noted that the definition of block sparse here is different from the conventional definition of block sparse representation for one-dimensional signal. For the latter, the data is distributed in adjacent data segments [41-43], which is not necessary at the situation of block sparsity based on tensor frame. In 2009, Eldar and Mishali introduced the concept of block sparsity in onedimensional signal [44]. For an underdetermined system $\boldsymbol{y}=\boldsymbol{D} \boldsymbol{x}$, assuming that the coefficient vector $\boldsymbol{x}$ consists of a series data segments with given length $\left\{d_{m}\right\}_{m=1}^{M}$ and denoting $m$-th segment as $\boldsymbol{x}[m]$, the segment is called block. Define the block sparse $l_{0}$-norm of $\boldsymbol{x}$ as $\|\boldsymbol{x}\|_{0, b}=$ $\sum_{m=1}^{M} f_{b}\left(\|\boldsymbol{x}[m]\|_{2}>0\right)$, where $\|\boldsymbol{x}[m]\|_{2}$ is the $l_{2}$ norm of the $m$-th data block; $f_{b}\left(\|\boldsymbol{x}[m]\|_{2}>0\right)$ is an indicator function which returns the value 1 if $\|x[m]\|_{2}>0$, otherwise returns 0 . If $\|\boldsymbol{x}\|_{0, b} \leq k, \boldsymbol{x}$ is called block $k$-sparse. Different from this definition of conventional block sparsity, the multi-linear block sparsity considers mainly the addition to the multi-linear structural restraint as interpreted in Definition 1.

It has been demonstrated that the recovery method based on Kronecker dictionary has much less strict requirements for coherence than the classic matching pursuit method with the same sparsity in regard to signal reconstruction, and it is deduced that the former has the higher successfully recovery bound $[28,45]$. In addition, by utilizing the structural information in each mode, the method based on tensor decomposition can perform robustly under the condition of high coherence [46].

\section{The proposed algorithm}

In higher-order signal processing, taking advantage of sparsity is becoming a research trend due to the curse of dimensionality problem [24, 47, 48]. Recently, applying CS scheme is rising in high-resolution radar imaging field, which can reconstruct the source signal from the undersampled measurements. The CS-based imaging method must solve an ill-posed linear inverse problem by using the sparsity of targets [12, 49-51]. However, for the large-scale data case, the classic $l_{0}$ algorithm has to encounter many computing problems. For instance, considering a scene of $1024 \times 1024$ observation points, the classic sparse reconstruction method must process the data up to 1,048,576 length, which is a large-scale linear system demanding numerous computational resources. A more efficient approach is investigated in this paper to ameliorate the above problems. As mentioned previously, the SAR imaging model maintains Kronecker structure and the scatterers in target scene are usually clustered together as blocks. As a result, we can take advantage of the block sparsity feature, building a sparse reconstruction SAR imaging algorithm based on Kronecker dictionary.

After decoupling procedure, the reflected signal can be formulated in the tensor form as explained in (8). Then, the nonzero scatter coefficients need to be recovered from the received signal $\mathcal{Y}$, i.e., finding a $\left(K_{1}, K_{2}, \ldots, K_{N}\right)$ block sparse representation corresponding to dictionaries $\boldsymbol{D}_{n}(n=1,2, \ldots, N)$. According to the compressed sampling theory, only a few rows need to be randomly extracted from the dictionary.

To begin with, we adopt a preprocessing approach for constructing the dictionaries from observation data. Let $\mathcal{E}=\mathcal{Y}-\mathcal{S} \times{ }_{1} D_{1} \times{ }_{2} D_{2} \times{ }_{3} \ldots \times{ }_{N} D_{N}$, then we have

$$
\begin{aligned}
\|\mathcal{E}\|_{F}^{2}= & \|\mathcal{Y}\|_{F}^{2}+\|\mathcal{S}\|_{F}^{2} \\
& -2\left\langle\mathcal{Y} \times{ }_{1} D_{1}^{T} \times{ }_{2} D_{2}^{T} \times{ }_{3} \ldots \times{ }_{N} D_{N}^{T}, \mathcal{S}\right\rangle ;
\end{aligned}
$$

thus, (7) can be rewritten as

$$
\max _{\left\{\boldsymbol{D}_{n}\right\}_{n=1}^{N}}\left\|\mathcal{Y} \times{ }_{1} \boldsymbol{D}_{1}^{T} \times{ }_{2} \boldsymbol{D}_{2}^{T} \times{ }_{3} \ldots \times \times_{N} \boldsymbol{D}_{N}^{T}\right\|_{F}^{2} .
$$

By utilizing alternating least squares (ALS) algorithm, (12) can be solved in an iterative scheme. Such algorithm fixes $(N-1)$ factor matrices in each iteration and updates the remaining component via least square approach, then repeats this process until the condition of convergence is satisfied.

For instance, fixing $\left\{\boldsymbol{D}_{n}\right\}_{n=2}^{N}$ first, the estimation of $\boldsymbol{D}_{1}$ can be obtained:

$$
\hat{\boldsymbol{D}}_{1}=\max _{\boldsymbol{D}_{1}}\left\|\boldsymbol{D}_{1}^{T} \boldsymbol{Z}_{(1)}\right\|_{F}^{2},
$$

where $\mathcal{Z}=\mathcal{Y} \times{ }_{2} \boldsymbol{D}_{2}^{T} \times \times_{3} \ldots \times_{N} \boldsymbol{D}_{N}^{T}$. Applying SVD on $Z_{(1)}$ and ensuring that the singular values are arranged in descending order, then the first $M_{1}$ left singular vectors of $\boldsymbol{Z}_{(1)}$ give the estimation of $\boldsymbol{D}_{1}$ [52]. Next, fixing $\left\{\boldsymbol{D}_{n}\right\}_{n=3}^{N}$ and $\boldsymbol{D}_{1}$, the estimation of $\boldsymbol{D}_{2}$ can be obtained in 
the same way. Repeat this procedure until all dictionaries are obtained.

Then, let $\boldsymbol{B}_{n}=\boldsymbol{D}_{n}\left(:, \mathscr{I}_{n}\right), n=1,2, \ldots, N$, then the received signal model can be rewritten as

$$
\hat{\boldsymbol{y}}=\left(\boldsymbol{B}_{N} \otimes \boldsymbol{B}_{N-1} \otimes \ldots \otimes \boldsymbol{B}_{1}\right) \boldsymbol{s}_{n z},
$$

where $\boldsymbol{s}_{n z} \in \mathbb{R}^{K}$ denotes the vector of nonzero coefficients. As a consequence, the solution of the problem can be presented as

$$
\boldsymbol{s}_{n z}=\underset{\boldsymbol{s}}{\arg \min }\left\|\left(\boldsymbol{B}_{N} \otimes \boldsymbol{B}_{N-1} \otimes \ldots \otimes \boldsymbol{B}_{1}\right) \boldsymbol{s}-\boldsymbol{y}\right\|_{2}^{2} .
$$

If $\boldsymbol{B}=\boldsymbol{B}_{N} \otimes \boldsymbol{B}_{N-1} \otimes \ldots \otimes \boldsymbol{B}_{1}$, then the solution of (15) can be deduced as $\boldsymbol{s}_{n z}=\left(\boldsymbol{B}^{T} \boldsymbol{B}\right)^{-1} \boldsymbol{B}^{T} \boldsymbol{y}$. For simplicity, assume that $K_{n}=K_{0}, n=1,2, \ldots, N$, then a sparse representation can be guaranteed if $\left(K_{0} \mu(\boldsymbol{D})\right)^{N}<2-\left(1+\left(K_{0}-1\right) \mu(\boldsymbol{D})\right)^{N}[28]$, where $\boldsymbol{D}=$ $\left(\boldsymbol{D}_{N} \otimes \boldsymbol{D}_{N-1} \otimes \ldots \otimes \boldsymbol{D}_{1}\right)$.

In addition, a more efficient calculation step can be employed. The relationship between $\mathcal{S}_{n z}$ and $\mathcal{Y}$ can be described as

$\mathcal{S}_{n z} \times{ }_{1}\left(\boldsymbol{B}_{1}^{T} \boldsymbol{B}_{1}\right) \times{ }_{2} \cdots \times{ }_{N}\left(\boldsymbol{B}_{N}^{T} \boldsymbol{B}_{N}\right)=\mathcal{Y} \times{ }_{1} \boldsymbol{B}_{1}^{T} \times{ }_{2} \cdots \times{ }_{N} \boldsymbol{B}_{N}^{T}$,

where $\mathcal{S}_{n z}$ is the tensor reshaping form of $\boldsymbol{s}_{n z}$. Defining $\mathcal{Q}=\mathcal{S}_{n z} \times{ }_{1} \boldsymbol{I} \times{ }_{2}\left(\boldsymbol{B}_{2}^{T} \boldsymbol{B}_{2}\right) \times_{3} \cdots \times_{N}\left(\boldsymbol{B}_{N}^{T} \boldsymbol{B}_{N}\right), \mathcal{R}=$ $\mathcal{Y} \times{ }_{1} \boldsymbol{B}_{1}^{T} \times{ }_{2} \cdots \times_{N} \boldsymbol{B}_{N}^{T}$, then we have $\boldsymbol{B}_{1}^{T} \boldsymbol{B}_{1} \boldsymbol{Q}_{(1)}=\boldsymbol{R}_{(1)}$. Because $\boldsymbol{B}_{1}^{T} \boldsymbol{B}_{1}$ is a self-adjoint matrix, there exists a fast algorithm which can obtain the solution of $\mathcal{Q}$ by utilizing Cholesky decomposition [53]. Next, unfold $\mathcal{Q}$ to a rectangular matrix along mode-2:

$$
\begin{array}{r}
\boldsymbol{Q}_{(2)}=\left(\boldsymbol{B}_{2}^{T} \boldsymbol{B}_{2}\right) \boldsymbol{S}_{n z(2)}\left[\left(\boldsymbol{B}_{N}^{T} \boldsymbol{B}_{N}\right) \otimes\left(\boldsymbol{B}_{N-1}^{T} \boldsymbol{B}_{N-1}\right)\right. \\
\left.\otimes \cdots \otimes\left(\boldsymbol{B}_{3}^{T} \boldsymbol{B}_{3}\right) \otimes \boldsymbol{I}\right]
\end{array}
$$

by defining $\mathcal{W}=\mathcal{S}_{n z} \times{ }_{1} \boldsymbol{I} \times{ }_{2} \boldsymbol{I} \times \times_{3}\left(\boldsymbol{B}_{3}^{T} \boldsymbol{B}_{3}\right) \times_{4} \cdots \times_{N}$ $\left(\boldsymbol{B}_{N}^{T} \boldsymbol{B}_{N}\right)$, similar to the above analysis, then we have $\boldsymbol{B}_{2}^{T} \boldsymbol{B}_{2} \boldsymbol{W}_{(2)}=\boldsymbol{Q}_{(2)}$. The solution of $\mathcal{W}$ can be found by utilizing Cholesky decomposition likewise. In accordance with this scheme, after $N$ steps, the estimation of $\mathcal{S}_{n z}$ will be obtained.

The algorithm flow is shown in Algorithm 1. During iterations, it is necessary to guarantee that the nonzero elements of core tensor are concentrated within a subset of index. When the convergence criterion is achieved, all nonzero elements and the corresponding index set can be obtained as the results, and the expected scatter coefficients will be recovered as well.

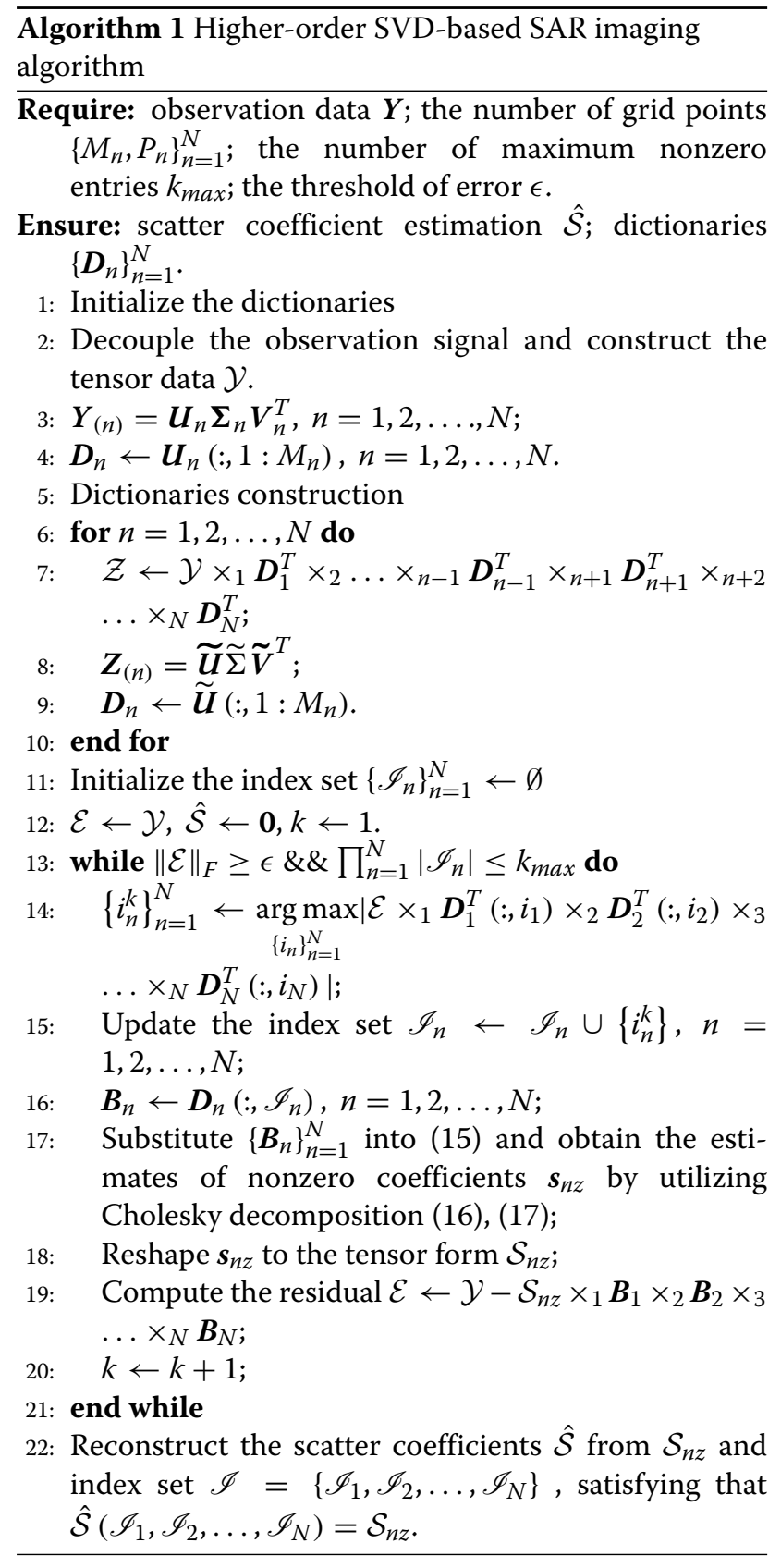

The key step of Algorithm 1 is estimating the nonzero coefficients by least squares procedure, which needs no more than $\left(2 N k\left(k^{N}+P+1\right)+N k^{2}\right)$ operations. By contrast, the orthogonal matching pursuit (OMP) algorithm [38, 54] needs $k^{N}\left(P^{N}+3 k^{N}\right)$ operations in this step. And in the residual update step, it is required to calculate $\mathcal{S}_{n z} \times \times_{1} \boldsymbol{B}_{1} \times{ }_{2} \boldsymbol{B}_{2} \times \times_{3} \ldots \times_{N} \boldsymbol{B}_{N}$ and subtract it from $\mathcal{Y}$, which needs $P^{N}(2 k+1)$ operations for the present algorithm, whereas the requirement for OMP algorithm is $P^{N}\left(2 k^{N}+1\right)$. Consequently, for a multi-linear $\left(K_{0}, K_{0}, \ldots, K_{0}\right)$-block sparse reconstruction problem described in Section 3, the maximum number 
of iteration of the present algorithm is $N K_{0}$. By contrast, OMP algorithm requires $K_{0}^{N}$ iterations for the same condition. The complexity analysis results indicate that the present algorithm can be used to estimate the scatter coefficients with much lower computational resource requirement compared with classic matching pursuit algorithm.

\section{Experiments and discussion}

This section will provide several numerical experiments to verify the effectiveness and performance of the proposed algorithm. The experiments include two parts: (1) SAR imaging realizations for ideal point scattering model and practical measured data and (2) performance comparisons between the proposed algorithm and the existing algorithms, including PFA [55, 56], OMP [38], and CoSaMP [57]. The PFA method is a kind of classic SAR imaging method which has good real-time response, although susceptible to the sidelobe effect. OMP and CoSaMP are both sparse reconstruction algorithms which can achieve the super-resolution performance, but they need to pay much more computational cost. The hardware configuration for the simulation system is Intel Core i7-5500U $2.4 \mathrm{GHz}$ CPU, 8GB RAM, Windows 10 OS.

\subsection{SAR imaging realizations}

Two groups of numerical experiments are demonstrated in this section. One tests the SAR imaging for ideal point targets, and the other investigates the effectiveness of realworld conditions. The simulation scenario is configured as follows: the center frequency is $9 \mathrm{GHz}$; the bandwidth is $1 \mathrm{GHz}$; the frequency resolution is $0.01 \mathrm{GHz}$; the angle aperture is $5^{\circ}$; the angle resolution is $0.05^{\circ}$; the sampling rate is $50 \%$.

\subsubsection{Ideal point targets}

Different SNRs Firstly, the imaging results under different SNRs are presented to confirm the feasibility of the proposed algorithm. Twenty ideal point scatterers are deployed in this simulation scenario, located along the diagonal line of the target scene randomly and clustered as three blocks. Corresponding to the settings of the SNR as 3 and $30 \mathrm{~dB}$, the SAR imaging realizations are shown in Fig. 2.

The simulations under different SNRs show the imaging qualities of the proposed algorithm and the reference algorithms. When SNR is low, the imaging results of classic matching pursuit algorithms exhibit distinct outliers, and PFA shows severe noisy ambiguity at the same time. As a contrast, the proposed algorithm performs much better under the low SNR condition. When SNR is high, the results of all methods indicate the congruent imaging quality.

Different number of scatterers In this simulation scenario, we consider the influence caused by the number of scatterers. The configuration is the same as the previous section, other than fixing the SNR at $10 \mathrm{~dB}$. When the number of scatterers is set to 30 and 150, the SAR imaging realizations are shown in Fig. 3.

It is shown from the simulations that when the number of scatterers is not large, all the methods are able to achieve an acceptable imaging resolution; contrastively, the proposed algorithm can obtain a neater imaging result. However, the scatterers located near the edge of the scene may obscure as the number of scattering points is increasing. The reconstructed scene for the PFA method show more obvious image noise than the matching pursuit algorithms, and the proposed algorithm can achieve the most accurate images under the same condition.

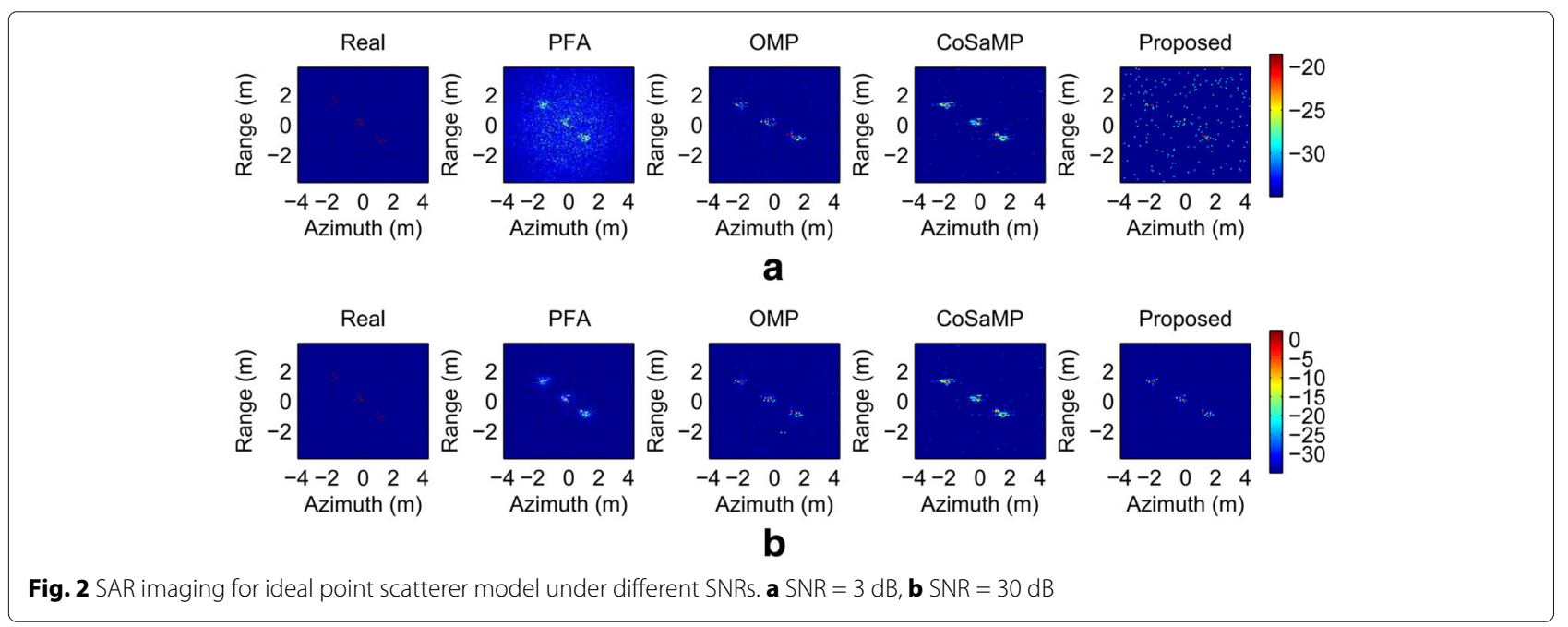



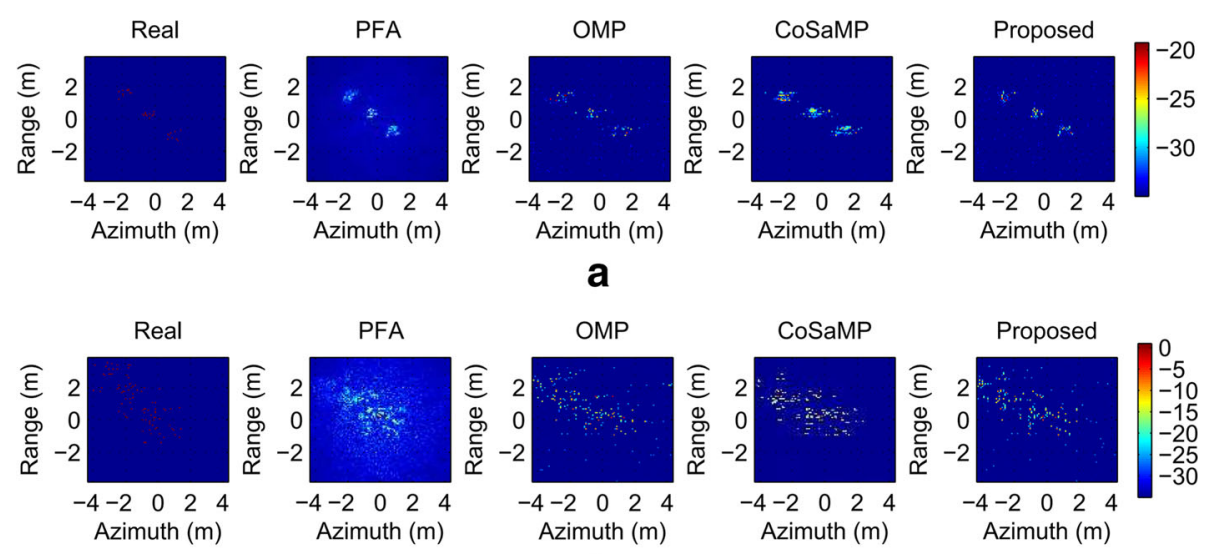

b

Fig. 3 SAR imaging for ideal point scatterer model under different number of scatterers. a 30 scatterers. b 150 scatterers

\subsubsection{Practical measured data}

In this part, a set of experiments based on practical measured data are demonstrated to verify the practicability of the proposed algorithm. The observation target is a crawler-type vehicle. There are three observation angles of a spotlight SAR:

- $77.5^{\circ} \sim 82.5^{\circ}$, the central angle is $80^{\circ}$

- $87.5^{\circ} \sim 92.5^{\circ}$, the central angle is $90^{\circ}$

- $97.5^{\circ} \sim 102.5^{\circ}$, the central angle is $100^{\circ}$

The center frequency and bandwidth are 9 and $1 \mathrm{GHz}$. As the results, when the central angles are $80^{\circ}, 90^{\circ}$, and $100^{\circ}$, the SAR imaging realizations are shown in Figs. 4, 5, and 6 , respectively.
The imaging results show the availability and superiority of the proposed algorithm in real SAR imaging. It is demonstrated that all the sparse reconstruction methods can achieve the higher resolution images than PFA. The latter can merely obtain fuzzy images, especially when the observation angle is not perpendicular to the target scene. Although OMP and CoSaMP are able to achieve the high-resolution results, there exist some problems with these classic matching pursuit techniques. For instance, when the observation angle is $90^{\circ}$, there is a distinct bright streak that appears on the edge of the target in the imaging result of CoSaMP. As indicated by the arrows in Figs. 4 and 6, CoSaMP shows raster lines over the target image at the central region, which will severely influence the recognition of target. In addition, OMP may result in
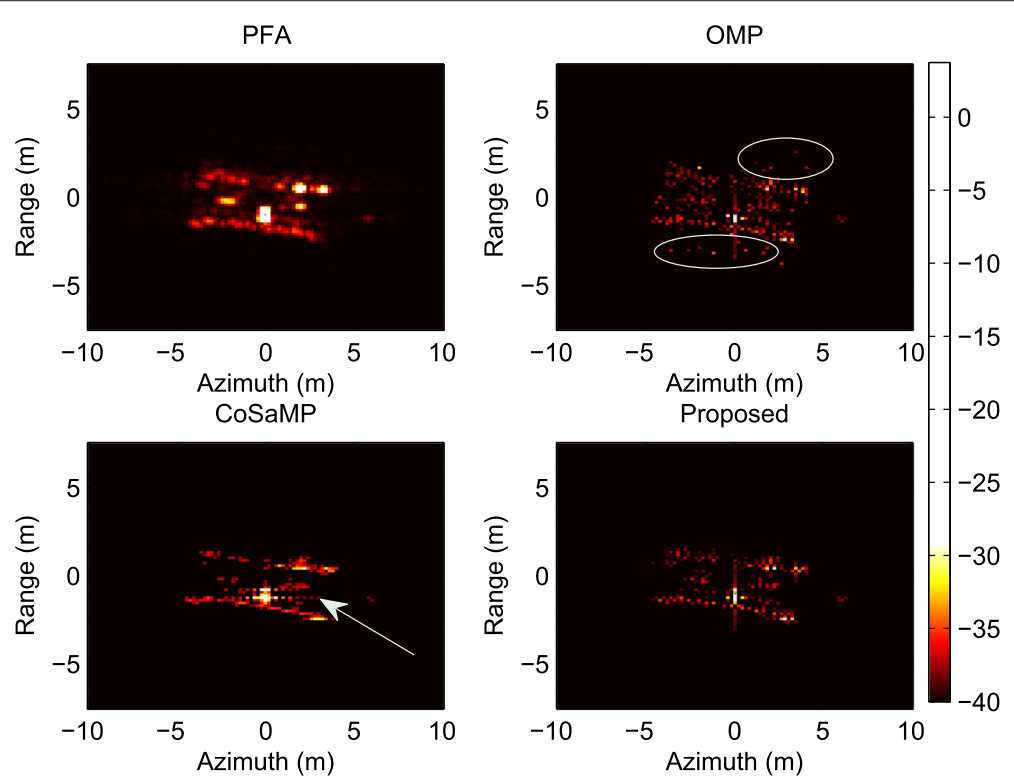

Fig. 4 SAR imaging for practical measured data, $\phi=80^{\circ}$ 

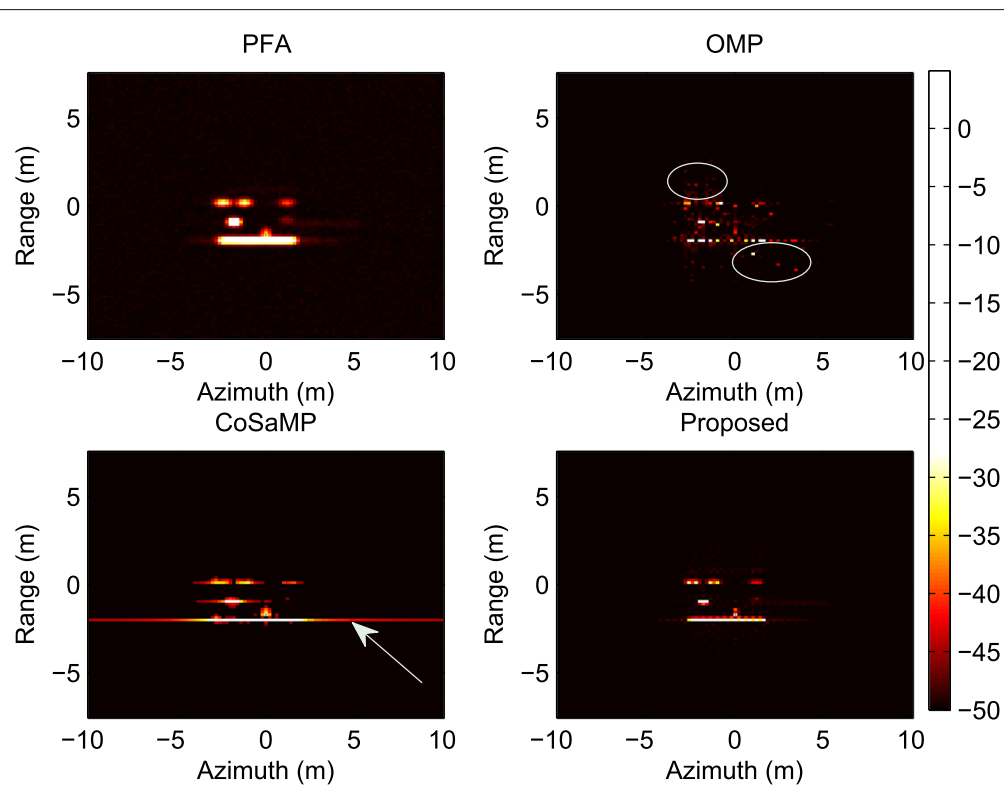

Fig. 5 SAR imaging for practical measured data, $\phi=90^{\circ}$

outliers on the sides of the target, which are marked by white circles in each images. In comparison, the proposed algorithm overcomes such defects and performs much better under the same scenario.

\subsection{Performance comparison}

Two performance indicators, root mean square error (RMSE) and computational cost, are discussed for both the proposed algorithm and the reference algorithms in this section.

\subsubsection{RMSE}

The RMSE of spotlight SAR model is defined as

$$
\mathrm{RMSE}=\sqrt{\frac{1}{L} \sum_{l=1}^{L} \frac{\left\|\boldsymbol{y}-\boldsymbol{D} \hat{\boldsymbol{s}}_{l}\right\|_{2}^{2}}{\|\boldsymbol{y}\|_{2}^{2}}}
$$

where $\hat{\boldsymbol{s}}_{l}$ denotes the estimate of scatter coefficient in the $l$-th Monte-Carlo independent trial. The configuration of this experiment is the same as Section 5.1.1. Two set of
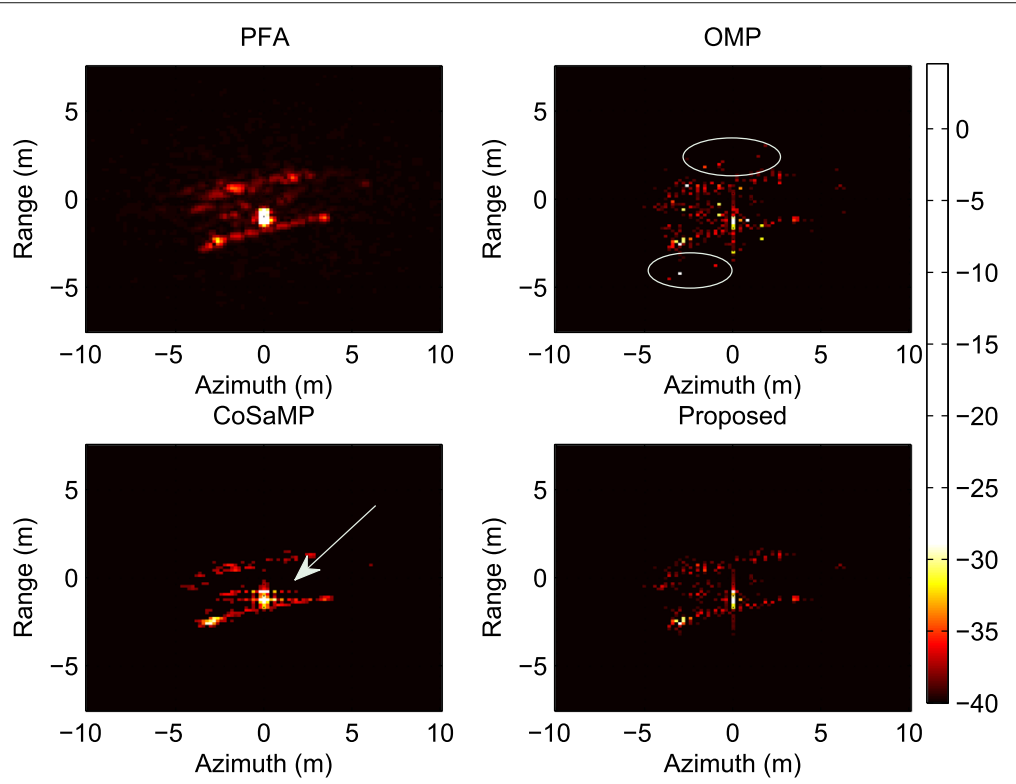

Fig. 6 SAR imaging for practical measured data, $\phi=100^{\circ}$ 


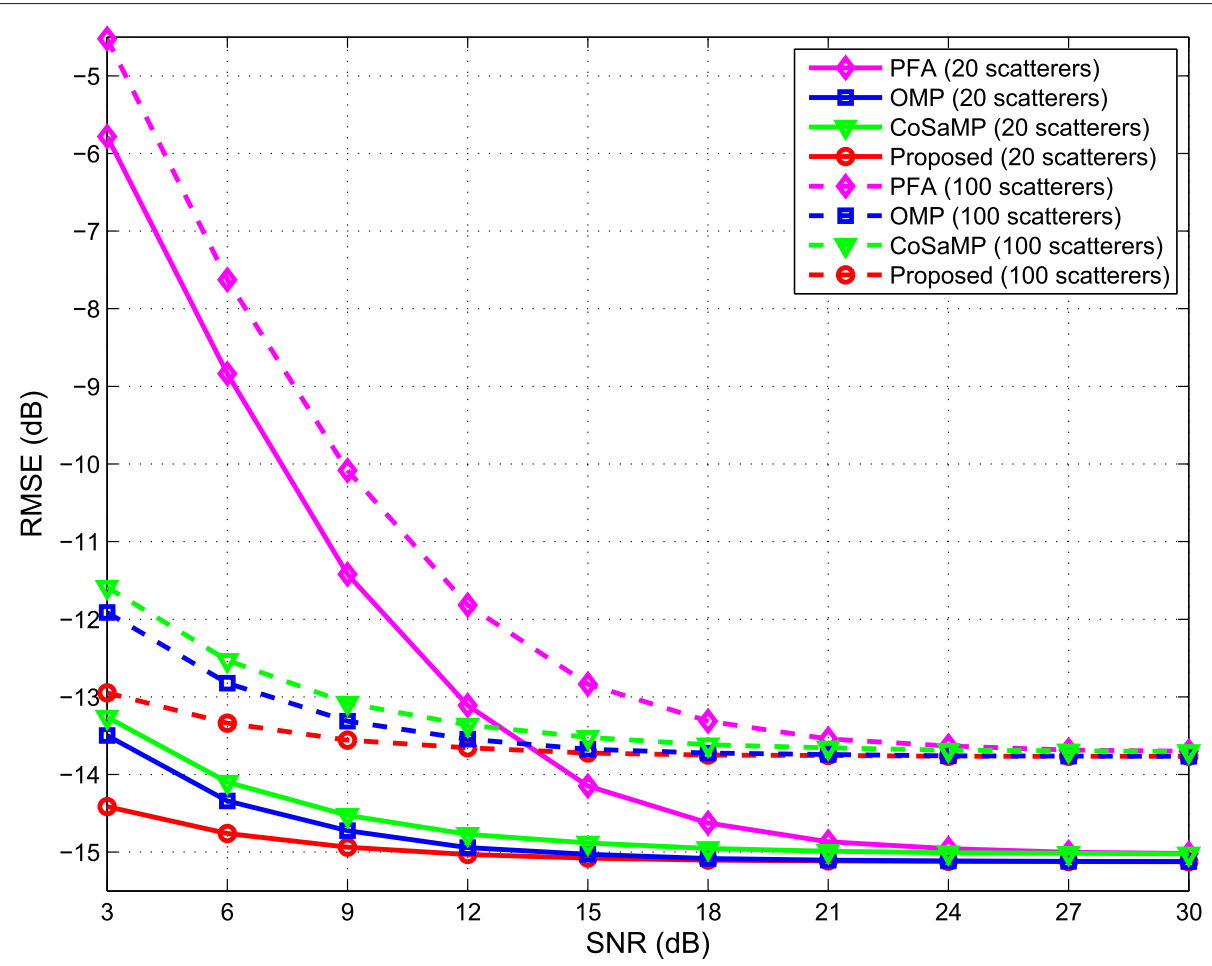

Fig. 7 RMSE comparison for different number of scatterers versus SNRs, by 500 Monte-Carlo trials

simulations for different number of scatterers are implemented. The SNR range is $3 \sim 30 \mathrm{~dB}$; the numbers of independent trials $L$ is set to 500 . The RMSE curves are shown in Fig. 7, where the solid lines and dashed lines indicate the experimental scenarios for 20 scatterers and 100 scatterers, respectively.

The simulation results indicate that the proposed algorithm performs superiorly compared with the reference methods for both the cases about the small and large number of scatterers. It can be seen that even if the SNR is low, the performances of sparse reconstruction schemes are more robust than conventional PFA method. As a result of the sensitivity to noise, the RMSE curve of PFA shows conspicuous precipitous variation as the SNR is less than $18 \mathrm{~dB}$. Considering the classic matching pursuit methods, since there are merely $2 k_{\max }$ atoms to be chosen in each iteration, CoSaMP runs faster than OMP for SAR imaging, which is indicated by the statistical results of the

Table 1 Average running time of imaging algorithms versus different SNRs

\begin{tabular}{lllll}
\hline Time $(\mathrm{s})$ & $\mathrm{SNR}=3 \mathrm{~dB}$ & $\mathrm{SNR}=12 \mathrm{~dB}$ & $\mathrm{SNR}=21 \mathrm{~dB}$ & $\mathrm{SNR}=30 \mathrm{~dB}$ \\
\hline PFA & 0.01736 & 0.01658 & 0.01564 & 0.01279 \\
OMP & 498.83 & 459.18 & 458.61 & 450.89 \\
CoSaMP & 1.1292 & 1.0759 & 1.0303 & 0.9784 \\
Proposed & 0.03719 & 0.03332 & 0.03135 & 0.03005 \\
\hline
\end{tabular}

computational cost in Section 5.2.2. However, the simulation results for RMSE show that the performance of CoSaMP is slightly worse than OMP. As a consequence, the existing SAR imaging methods are always difficult to achieve both the high speed and large success ratio of recovery. In contrast, by exploiting the multi-linear block sparsity of targets, the proposed algorithm can gain satisfactory performances on the both aspects.

\subsubsection{Computing resources requirement}

This section investigates the requirements for computational cost. The configuration of this part is the same as Section 5.1.1 and the evaluation is done in terms of CPU time. The statistical results of running time for the case of different SNRs are shown in Table 1.

For the case about different numbers of scatterers, set the configuration to be the same as Section 5.1.1. The statistical results of running time are shown in Table 2.

Table 2 Average running time of imaging algorithms versus different numbers of scatterers (indicated by $N_{s}$ )

\begin{tabular}{lllll}
\hline Time $(s)$ & $N_{s}=20$ & $N_{s}=50$ & $N_{s}=100$ & $N_{s}=150$ \\
\hline PFA & 0.01716 & 0.01832 & 0.02437 & 0.04121 \\
OMP & 519.67 & 525.62 & 537.96 & 568.34 \\
CoSaMP & 0.95787 & 0.96073 & 0.98114 & 1.2098 \\
Proposed & 0.04519 & 0.04651 & 0.04762 & 0.05133 \\
\hline
\end{tabular}


The statistical data of Tables 1 and 2 indicate that there exist noteworthy differences of computational cost among these algorithms. The fastest two algorithms are PFA and the proposed algorithm, followed by CoSaMP and OMP in sequence. Nevertheless, the PFA cannot provide robust performance, as the RMSE comparison in the previous section. On the other hand, although the classic matching pursuit methods have high-resolution performance, the requirements to computing resources are considerable, especially for OMP algorithm. In each iteration, OMP must match every atom, which results in a high time consumption. As a contrast, the proposed algorithm can obtain the optimal RMSE performance and achieve a running speed faster one order of magnitude than CoSaMP, which makes it suitable for the real-time applications.

\section{Conclusions}

In a practical scenario, the block sparse distribution of targets is a common assumption for SAR imaging. Nevertheless, the existing methods seldom take advantage of such geometrical feature. In this paper, a Kronecker constrained spotlight SAR imaging algorithm based on tensor decomposition is developed, which exploits the block sparsity of the target scene. HOSVD modeling of the decoupling signal received is employed by introducing a preprocessing scheme for dictionary construction. Then, a multi-linear sparse reconstruction algorithm is deduced to obtain the estimation of scatter coefficients. Since the proposed algorithm utilizes the structural information of targets, a more robust performance can be achieved for imaging quality. A group of numerical experiments based on practical measured data are presented to verify the effectiveness of the developed method. In addition, the complexity analysis indicates that the algorithm reduces the demand for computational cost comparing with the classic matching pursuit methods. The statistical results of simulations confirm the superiority of the present algorithm as well, which is vitally important in real-time applications of engineering technique.

\footnotetext{
Acknowledgements

This work was supported in part by the National Natural Science Foundation of China (NSFC) under Grant U1533125 and National Science and technology major project under Grant 2016ZX03001022. The authors would like to thank the anonymous reviewers for their valuable comments that significantly improved the quality of this paper.
}

\section{Authors' contributions}

Y-FG conceived and verified the feasibility of SAR imaging by introducing tensor modeling and developed the multi-linear sparse target reconstruction algorithm based on tensor decomposition; $Y$ - $F G, X-C C$, and $Y Y$ performed the experiments; $\mathrm{GG}$ and $\mathrm{QW}$ contributed to the design of numerical simulations; Y-FG wrote this paper; X-CC, YY, Y-BZ, and GG checked the manuscript and contributed to the rearrangement of the materials. All authors read and approved the final manuscript.

\section{Competing interests}

The authors declare that they have no competing interests.

\section{Publisher's Note}

Springer Nature remains neutral with regard to jurisdictional claims in published maps and institutional affiliations.

\section{Author details}

${ }^{1}$ School of Electronic Engineering, University of Electronic Science and Technology of China, No. 2006, Xiyuan Ave., West Hi-Tech Zone, Chengdu 611731, China. ${ }^{2}$ College of Telecommunication and Information Engineering, Nanjing University of Posts and Telecommunications, 66 Xinmofan Road, Nanjing 210003, China.

Received: 27 December 2016 Accepted: 19 May 2017

Published online: 12 June 2017

\section{References}

1. JC Curlander, RN MCDonough, Synthetic Aperture Radar: Systems and Signal Processing. (Wiley-Interscience, New York, 1991)

2. GC Walter, SG Ron, MM Ronald, Spotlight Synthetic Aperture Radar. (Artech House Publishers, Norwood, 1995)

3. G Franceschetti, R Lanari, Synthetic Aperture Radar Processing. (CRC press, Boca Raton, 1999)

4. A Marino, Synthetic Aperture Radar. (Springer, Berlin, 2012), pp. 9-27

5. M Soumekh, Synthetic Aperture Radar Signal Processing with MATLAB Algorithms. (Wiley-Interscience, New York, 1999)

6. E Mason, IY Son, B Yazici, Passive synthetic aperture radar imaging using low-rank matrix recovery methods. IEEE J. Sel. Topic Signal Process. 9(8), 1570-1582 (2015). doi:10.1109/JSTSP.2015.2465361

7. R Baraniuk, P Steeghs, in 2007 IEEE Radar Conference. Compressive radar imaging (IEEE, Boston, 2007), pp. 128-133

8. L Zhang, Qiao Z-j, M Xing, Y Li, Z Bao, High-resolution isar imaging with sparse stepped-frequency waveforms. IEEE Trans. Geosci. Remote Sens. 49(11), 4630-4651 (2011)

9. L Zhang, Z-J Qiao, M-D Xing, J-L Sheng, R Guo, Z Bao, High-resolution isar imaging by exploiting sparse apertures. IEEE Trans. Antennas Propag. 60(2), 997-1008 (2012)

10. B Sun, Y Cao, J Chen, C Li, Z Qiao, Compressive sensing imaging for general synthetic aperture radar echo model based on Maxwell's equations. EURASIP J. Adv. Signal Process. 2014(1), 153 (2014). doi:10.1186/1687-6180-2014-153

11. G Li, Q Hou, $S$ Xu, Z Chen, Multi-target simultaneous isar imaging based on compressed sensing. EURASIP J. Adv. Signal Process. 2016(1), 27 (2016). doi:10.1186/s13634-016-0327-1

12. Z Zhang, Y Xu, J Yang, $X \mathrm{Li}$, D Zhang, A survey of sparse representation: algorithms and applications. IEEE Access. 3, 490-530 (2015). doi:10.1109/ACCESS.2015.2430359

13. B Chen, J Wang, H Zhao, N Zheng, JC Príncipe, Convergence of a fixed-point algorithm under maximum correntropy criterion. IEEE Signal Process. Lett. 22(10), 1723-1727 (2015)

14. W Ma, H Qu, G Gui, L Xu, J Zhao, B Chen, Maximum correntropy criterion based sparse adaptive filtering algorithms for robust channel estimation under non-gaussian environments. J. Frankl. Inst. 352(7), 2708-2727 (2015)

15. J Fang, Z Xu, B Zhang, W Hong, Y Wu, Fast compressed sensing SAR imaging based on approximated observation. IEEE J. Sel. Topic Appl. Earth Obs. Remote Sens. 7(1), 352-363 (2014)

16. L Zhang, J Duan, Z-J Qiao, M-D Xing, Z Bao, Phase adjustment and ISAR imaging of maneuvering targets with sparse apertures. IEEE Trans. Aerosp. Electron. Syst. 50(3), 1955-1973 (2014)

17. J Zhang, Y Ban, D Zhu, G Zhang, Random filtering structure-based compressive sensing radar. EURASIP J. Adv. Signal Process. 2014(1), 94 (2014). doi:10.1186/1687-6180-2014-94

18. X Zhang, G Liao, S Zhu, D Yang, W Du, Efficient compressed sensing method for moving-target imaging by exploiting the geometry information of the defocused results. IEEE Geosci. Remote Sens. Lett. 12(3), 517-521 (2015)

19. X Cong, G Gui, X Li, G Wen, X Huang, Q Wan, Object-level sar imaging method with canonical scattering characterisation and inter-subdictionary interferences mitigation. IET Radar Sonar Navig. 10(4), 784-790 (2016)

20. L Zhang, H Wang, Qiao Z-j, Resolution enhancement for isar imaging via improved statistical compressive sensing. EURASIP J. Adv. Signal Process. 2016(1), 80 (2016). doi:10.1186/s13634-016-0379-2 
21. L Zhao, L Wang, G Bi, S Li, L Yang, H Zhang, Structured sparsity-driven autofocus algorithm for high-resolution radar imagery. Signal Process. 125, 376-388 (2016)

22. R Bro, Parafac. tutorial and applications. Chemometr. Intell. Lab. Syst. 38(2), 149-171 (1997)

23. ND Sidiropoulos, R Bro, GB Giannakis, Parallel factor analysis in sensor array processing. IEEE Trans. Signal Process. 48(8), 2377-2388 (2000)

24. A Cichocki, D Mandic, L De Lathauwer, G Zhou, Q Zhao, C Caiafa, HA Phan, Tensor decompositions for signal processing applications: from two-way to multiway component analysis. IEEE Signal Process. Mag. 32(2), 145-163 (2015)

25. L-H Lim, P Comon, Multiarray signal processing: tensor decomposition meets compressed sensing. Comptes Rendus Mecanique. 338(6), 311-320 (2010)

26. MF Duarte, YC Eldar, Structured compressed sensing: from theory to applications. IEEE Trans. Signal Process. 59(9), 4053-4085 (2011)

27. ND Sidiropoulos, A Kyrillidis, Multi-way compressed sensing for sparse low-rank tensors. IEEE Signal Process. Lett. 19(11), 757-760 (2012)

28. CF Caiafa, A Cichocki, Multidimensional compressed sensing and their applications. Wiley Interdiscip. Rev. Data Min. Knowl. Disc. 3(6), 355-380 (2013). doi:10.1002/widm.1108

29. S Friedland, Q Li, D Schonfeld, Compressive sensing of sparse tensors. IEEE Trans. Image Process. 23(10), 4438-4447 (2014). doi:10.1109/TIP.2014.2348796

30. E Li, MJ Shafiee, F Kazemzadeh, A Wong, Sparse reconstruction of compressive sensing multi-spectral data using an inter-spectral multi-layered conditional random field model. IEEE Access. 4, 5540-5554 (2016). doi:10.1109/ACCESS.2016.2598320

31. M Haardt, F Roemer, G Del Galdo, Higher-order SVD-based subspace estimation to improve the parameter estimation accuracy in multidimensional harmonic retrieval problems. IEEE Trans. Signal Process. 56(7), 3198-3213 (2008)

32. F Roemer, Advanced algebraic concepts for efficient multi-channel signal processing. (PhD thesis, Ilmenau University of Technology, IImenau, 2013)

33. TG Kolda, BW Bader, Tensor decompositions and applications. SIAM Rev. 51(3), 455-500 (2009). doi:10.1137/07070111X

34. DH Vu, Advanced techniques for synthetic aperture radar image reconstruction. (PhD thesis, University of Florida, Gainesville, 2012)

35. W Xu, P Huang, Y Deng, Efficient sliding spotlight SAR raw signal simulation of extended scenes. EURASIP J. Adv. Signal Process. 2011(1), 52 (2011). doi:10.1186/1687-6180-2011-52

36. L Zhang, Li H-I, Qiao Z-j, Xing M-d, Z Bao, Integrating autofocus techniques with fast factorized back-projection for high-resolution spotlight SAR imaging. IEEE Geosci. Remote Sens. Lett. 10(6), 1394-1398 (2013)

37. L Greengard, J Lee, Accelerating the nonuniform fast fourier transform. SIAM Rev. 46(3), 443-454 (2004)

38. JA Tropp, Greed is good: algorithmic results for sparse approximation. IEEE Trans. Inf. Theory. 50(10), 2231-2242 (2004)

39. S Jokar, V Mehrmann, Sparse solutions to underdetermined Kronecker product systems. Linear Algebra Appl. 431(12), 2437-2447 (2009)

40. S Jokar, in 2010 44th Annual Conference on Information Sciences and Systems (CISS). Sparse recovery and kronecker products, (2010), pp. 1-4. doi:10.1109/CISS.2010.5464722

41. YC Eldar, M Mishali, in 2009 16th International Conference on Digital Signal Processing. Block sparsity and sampling over a union of subspaces, (2009), pp. 1-8. doi:10.1109/ICDSP.2009.5201211

42. YC Eldar, P Kuppinger, H Bolcskei, Block-sparse signals: uncertainty relations and efficient recovery. IEEE Trans. Signal Process. 58(6), 3042-3054 (2010)

43. RG Baraniuk, V Cevher, MF Duarte, C Hegde, Model-based compressive sensing. IEEE Trans. Inf. Theory. 56(4), 1982-2001 (2010)

44. YC Eldar, M Mishali, Robust recovery of signals from a structured union of subspaces. IEEE Trans. Inf. Theory. 55(11), 5302-5316 (2009). doi:10.1109/TIT.2009.2030471

45. MF Duarte, RG Baraniuk, Kronecker compressive sensing. IEEE Trans. Image Process. 21(2), 494-504 (2012)

46. Y-F Gao, L Zou, Q Wan, A two-dimensional arrival angles estimation for I-shaped array based on tensor decomposition. AEU - Int. J. Electron. Commun. 69(4), 736-744 (2015). doi:10.1016/j.aeue.2015.01.001

47. DL Donoho, et al., High-dimensional data analysis: the curses and blessings of dimensionality. AMS Math Chall. Lect., 1-32 (2000)
48. IV Oseledets, EE Tyrtyshnikov, Breaking the curse of dimensionality, or how to use SVD in many dimensions. SIAM J. Sci. Comput. 31(5), 3744-3759 (2009)

49. B Sun, H Gu, M Hu, Z Qiao, in SPIE Sensing Technology+ Applications. Compressive sensing for a general sar imaging model based on maxwell's equations (International Society for Optics and Photonics, San Diego, 2015), pp. 948402-948402

50. Y Li, Y Wang, Sparse SM-NLMS algorithm based on correntropy criterion. Electron. Lett. 52(17), 1461-1463 (2016)

51. Y Li, C Zhang, S Wang, Low-complexity non-uniform penalized affine projection algorithm for sparse system identification. Circ. Syst. Signal Process. 35(5), 1611-1624 (2016)

52. L De Lathauwer, B De Moor, J Vandewalle, On the best rank-1 and rank$(r 1, r 2, \ldots, r n)$ approximation of higher-order tensors. SIAM J. Matrix Anal. Appl. 21(4), 1324-1342 (2000)

53. R Rubinstein, M Zibulevsky, M Elad, Efficient implementation of the K-SVD algorithm using batch orthogonal matching pursuit. Technical Report 8, Computer Science Department, Technion - Israel Institute of Technology (2008)

54. JA Tropp, SJ Wright, Computational methods for sparse solution of linear inverse problems. Proc. IEEE. 98(6), 948-958 (2010)

55. Y Yuan, J Sun, S Mao, PFA algorithm for airborne spotlight SAR imaging with nonideal motions. IEE Proc. Radar Sonar Navig. 149(4), 174-182 (2002)

56. BD Rigling, RL Moses, Polar format algorithm for bistatic SAR. IEEE Trans. Aerosp. Electron. Syst. 40(4), 1147-1159 (2004)

57. D Needell, JA Tropp, Cosamp: Iterative signal recovery from incomplete and inaccurate samples. Appl. Comput. Harmon. Anal. 26(3), 301-321 (2009)

\section{Submit your manuscript to a SpringerOpen ${ }^{\circ}$ journal and benefit from:}

- Convenient online submission

- Rigorous peer review

- Open access: articles freely available online

- High visibility within the field

- Retaining the copyright to your article

Submit your next manuscript at springeropen.com 